\title{
Brownian dynamics simulation of the crystallization dynamics of charged colloidal particles
}

\author{
Lingyun $\mathrm{Gu}^{\mathrm{a}, \mathrm{b}}$, Shenghua $\mathrm{Xu}{ }^{\mathrm{a}, \mathrm{b}}$, Zhiwei Sun ${ }^{\mathrm{a}, \mathrm{b}, *}$, Jin Tong Wang ${ }^{\mathrm{c}}$ \\ ${ }^{a}$ Key Laboratory of Microgravity, Institute of Mechanics, Chinese Academy of Sciences, 100190 Beijing, People's Republic of China \\ ${ }^{\mathrm{b}}$ National Microgravity Laboratory, Institute of Mechanics, Chinese Academy of Sciences, 100190 Beijing, People's Republic of China \\ ${ }^{\mathrm{C}}$ Department of Physics, Southern University and AE'M College, Baton Rouge, LA 70813, USA
}

\section{A R T I C L E I N F O}

Article history:

Received 13 April 2010

Accepted 7 July 2010

Available online 11 July 2010

\section{Keywords:}

Colloidal crystallization

Brownian dynamics simulation

Yukawa potential

\begin{abstract}
A B S T R A C T
Crystal formation process of charged colloidal particles is investigated using Brownian dynamics (BD) simulations. The particles are assumed to interact with the pair-additive repulsive Yukawa potential. The time evolution of crystallization process and the crystal structure during the simulation are characterized by means of the radial distribution functions (RDF) and mean square displacement (MSD). The simulations show that when the interaction is featured with long-range, particles can spontaneously assemble into body-centered-cubic (BCC) arrays at relatively low particle number density. When the interaction is short-ranged, with increasing the number density particles become trapped into a stagnant disordered configuration before the crystallization could be actualized. The simulations further show that as long as the trapped configurations are bypassed, the face-centered-cubic (FCC) structures can be achieved and are actually more stable than BCC structures.
\end{abstract}

(c) 2010 Elsevier Inc. All rights reserved.

\section{Introduction}

To further explore the processes involved in crystal growth including the mechanisms of the growth, there are still enormous challenges ahead. Charged colloidal particles suspended in de-ionized water under appropriate conditions can be self-assembled into highly ordered arrays of particles: colloidal crystals $[1,2]$. This self-assembly process of colloids directly appears analogous to their atomic or molecular counterparts and therefore can provide a useful model system for studying general principles of the crystallization of materials. Since time and length scales in colloidal crystals are several orders of magnitude larger than those of atomic or molecular crystals, one can acquire various more suitable tools and instruments for observation and measurement in investigation of crystallization to gain an insight into crystal growth.

Understanding the mechanisms underlying transition dynamics of colloidal crystallization is of fundamental importance to assess the validity of classical crystal growth theories and to control crystal morphology. Much recent work has been focused on colloidal interactions and phase transitions [3,4]. The spatial ordering of colloidal particles has been intensively studied and standard crystal growth theories are applied to explain the mechanisms of

\footnotetext{
* Corresponding author at: National Microgravity Laboratory, Institute of Mechanics, Chinese Academy of Sciences, 100190 Beijing, People's Republic of China. Fax: +86 1082544096

E-mail address: sunzw@imech.ac.cn (Z. Sun).
}

colloidal crystallization [5,6]. The computer simulations have contributed considerably to the investigation of the behavior of colloidal crystallization at the particle level. Monte Carlo (MC) simulation is capable of predicting the thermodynamic and structural properties of a system and it has been used to study the phase diagram of suspensions of charged colloids $[7,8]$. However, MC method is not suitable for providing the information of dynamic behavior, including the nucleation pathways. Molecular dynamics (MD) simulation has also been adopted to study the colloidal crystallization $[9,10]$. But it is not adaptable to simulate both colloidal particles and solvent particles motion, because the timescale characterizing the motions of the colloidal particles and the solvent particles can differ by several orders of magnitude. To solve this problem, the colloidal particles are taken as MD particles, and the force of solvent particles is ignored for such MD simulation [10], in which the system is quenched and super-cooled and the temperature rescaling method is applied. On the other hand, the Brownian dynamics (BD) technique, as a mesoscopic method, is more suitable for characterizing the motion of colloidal particles because of its ability to average out these fast modes of the solvent allows one to simulate much larger time scales. Accordingly, the solvent molecules' impacts on the colloidal particles are modeled by a combination of random and frictional force terms. Therefore, BD method mimics the behavior of colloidal particles much more properly, and can provide a simplified and useful model of the colloidal particles motion. BD simulation has been used to study the two-dimensional colloidal systems and found new aspects of 
colloidal ordering process which are quite different from the classical nucleation theory [11]. However, two-dimensional model has its limitations in revealing the real complex three-dimensional world of crystal growth in colloidal systems, especially when dealing with spatial structures, dynamic process, etc.

In this paper, we present three-dimensional BD simulation for dynamic properties of charged colloidal crystallization. The results show that, BCC crystals are evolved spontaneously from a disordered, randomly dispersed system. But an ordered FCC structure is not directly accessible from a disordered state under the conditions in which a stable FCC state is expected. However, a transition from BCC to FCC structure could be achieved if the initial state is BCC. The reasons for the simulation results are also explained in detail in this study.

\section{Materials and methods}

In $\mathrm{BD}$ simulation, the governing equation of the motion of particles is the Langevin equation, and the diffusive motion of the particles is simulated by adding random displacements, proportional to the square root of the time step $[12,13]$. The algorithm for the particles motion in the simulation based on the Langevin equation is of the form,

$r(t+\Delta t)=r(t)+\frac{D}{k_{B} T} F(t) \Delta t+\Delta r^{G}$,

where the component $\Delta r^{G}$ is chosen from Gaussian distribution with zero mean.

In the simulation of this work, we consider a system composed of charged colloidal particles. Interactions between the colloidal particles are approximated by the repulsive Yukawa potential $[8,14]$, which is given by

$u(r)=\frac{\varepsilon}{r} \cdot \exp [-\kappa(r-d)]$

where $\varepsilon$ basically represents the amplitude of the Yukawa repulsion in units of $k_{\mathrm{B}} T$, and $k_{\mathrm{B}}$ denotes the Boltzmann constant. And $\kappa$ is the inverse screening length. The dependence of $u(r)$ on the two parameters, $\varepsilon$ and $\kappa$, is shown in Fig. 1 .

Yukawa potential has been widely used for modeling and analysis of charge-stabilized colloidal suspensions, with its parameters continuously tunable from Coulomb to hard-sphere-like interactions. For large $\kappa$, the potential is shorter ranged and approaches hard-sphere-like interactions. On the other hand, small $\kappa$ corresponds with long-range repulsion as well as a soft interaction. The total interaction energy is given by the sum of all pairs and thus has the form:

$U(r)=\sum_{i<j}^{N} u\left(r_{i j}\right)$.

For real systems, however, the interaction between any two charged particles is strongly influenced by other particles surrounding the two particles in consideration. These so-called screening effects have been directly observed in the experiments with the aid of optical tweezers by Brunner et al. [15] as well as our group [16,17]. When the distance between the two particles is larger than the average pair separation, more particles may come in between them, making the screening effects stronger, and thus the interaction between the two particles rapidly decays [18]. Therefore, Eq. (1) no longer holds at large inter-particle distance. To partially take these effects into consideration, $u(r)$ is set to be zero at larger distances, and this procedure is called "a truncated Yukawa potential with a density-dependent cut-off" [14]. Its a simple measure to manifest the screening effects in the computer simulations. In this way, the cutoff of the interaction between the particles is chosen with some
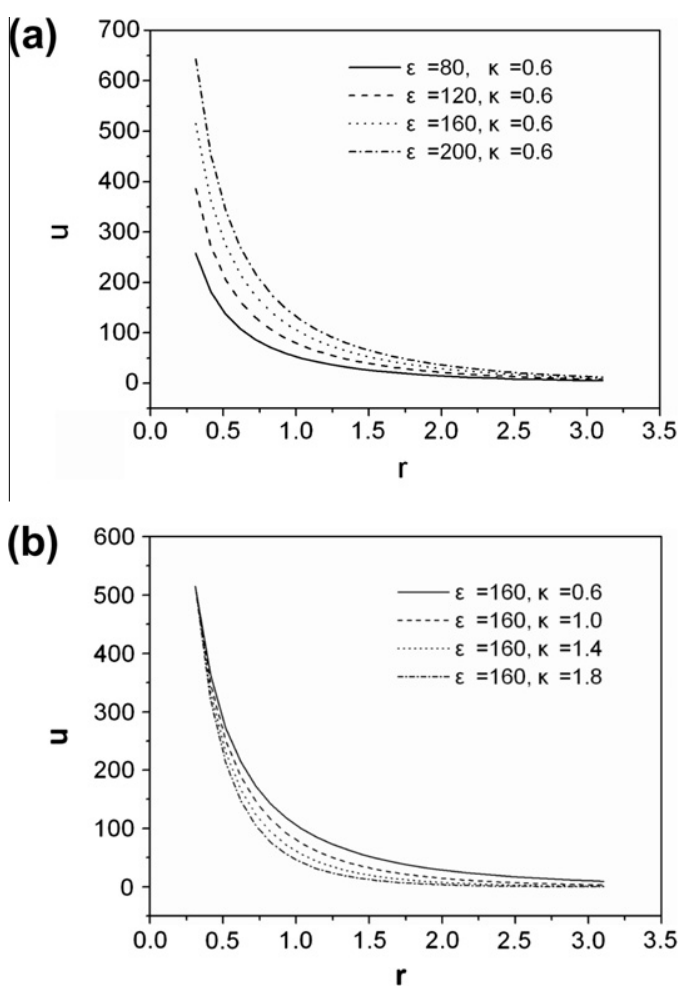

Fig. 1. Yukawa potential as a function of $\varepsilon(\mathrm{a})$ and $\kappa(\mathrm{b})$.

care. The reduced cut-off distance at large $\kappa$ as for long-ranged interactions was taken to be 3.045 in units of the relevant length scale in this study. This value is, to some extent, larger than that suggested in some previous studies [10], as the weekly screened system considered in this study requires a larger cutoff radius [18].

A cubic simulation box with periodic boundary conditions was used. The initial configuration was constructed by placing a number of particles randomly inside the three-dimensional simulation box. The simulation was carried out at constant volume.

The calculation parameters are set as follows: the simulation time step $\Delta t=2.0 \times 10^{-6} \mathrm{~s}$, the viscosity of the solvent $\eta=1.0 \times 10^{-3} \mathrm{~Pa} \mathrm{~s}$, the temperature $T=293.15 \mathrm{~K}$. The time step $\Delta t$ is much larger than the velocity relaxation time $\tau=m D / k_{\mathrm{B}} T=2.0 \times 10^{-10} \mathrm{~s}$, and much smaller than the diffusion time $\tau_{\mathrm{D}}=a^{2} / D=1.26 \times 10^{-4} \mathrm{~s}$, where the radius of the particle $a$ is $60 \mathrm{~nm}, D$ is the Stokes-Einstein diffusion coefficient which is given by,

$D=\frac{k_{B} T}{6 \pi \eta a}$

The relevant length scale is chosen to be

$L_{0}=\left(\frac{6 N}{\pi V}\right)^{-1 / 3}$,

where $N$ is the total number of the particles, and $V$ denotes for the system volume. Thus $N / V$ is the particle number density of the system. So the reduced parameters are as follows: $r$ is the radial distance between two particles, and $d$ is the particle diameter. The other units of the parameters are set as follows: temperature units $T_{0}=293.15 \mathrm{~K}$, energy units $u_{0}=k_{\mathrm{B}} T$, time units $t_{0}=L_{0}^{2} / D_{0}$. Thus the normalized parameters are $T^{*}=1, \varepsilon^{*}=\varepsilon / k_{\mathrm{B}} T$. The time step and diffusion coefficient are normalized together as $(D t)^{*}=D t / L_{0}^{2}$.

All the variables normalized above are summarized in Table 1.

A number of approaches, such as the structure factor [19], Lindemann parameter [20], and Bond-Order parameters [21] have been used to characterize crystallization. For instance, the 
Table 1

The normalization scheme of the simulation variables.

\begin{tabular}{lll}
\hline $\begin{array}{l}\text { Calculation } \\
\text { parameters }\end{array}$ & $\begin{array}{l}\text { Units of the } \\
\text { parameters }\end{array}$ & $\begin{array}{l}\text { The normalized } \\
\text { parameters }\end{array}$ \\
\hline$T=293.15 \mathrm{~K}$ & $T_{0}=293.15 \mathrm{~K}$ & $T^{*}=1$ \\
$\Delta t=2.0 \times 10^{-6} \mathrm{~S}$ & $u_{0}=k_{\mathrm{B}} T$ & $\varepsilon^{*}=\varepsilon / k_{\mathrm{B}} T$ \\
$a=60 \mathrm{~nm}$ & $t_{0}=L_{0}^{2} / D_{0}$ & $(D t)^{*}=D t / L_{0}^{2}$ \\
\hline
\end{tabular}

structure factor, which is the Fourier transformation of radial distribution functions (RDF), is a useful indicator in analyzing long-range order of the system. The method of Bond-Order parameters provides a useful approach to assessing local as well as global order structures. However, since in our simulations the structure's evolution did not clearly demonstrate a tendency that crystal growth extends from local to global structure and also random motions cause some fluctuations for the values of Bond-Order parameters, for simplicity and efficiency, the RDF method is adopted in this study. The methods of RDF and MSD are used together to determine the evolution of the system.

The method RDF was used to analyze the ordering process and distinguish the structure of the resulting crystals. The RDF is defined as

$\mathrm{RDF}=\frac{\rho(r)}{\rho}$.

Here $\rho$ is the average density of the system, while $\rho(r)$ is the local density at distance $r$ from a chosen "central" particle. RDF gives the probability of finding a particle in the distance $r$ from the central particle. A system that is in liquid state typically has a smooth RDF with a small number of peaks at short distances, and decay to one after the first peak at longer distances. In a system of crystal state, the RDF has many sharp peaks whose separations and heights are characteristic of the lattice structure. And the first peak denotes the position of the nearest neighbor of the particles. So RDF is useful to describe the structure of a system and thus can be adopted to measure the extent of the system's ordering.

The mean square displacement (MSD) measures the distance that a particle travels during the simulation process. MSD is calculated by aver aging over all the particles in the system and thus is given by

$\operatorname{MSD}=\left\langle r^{2}(t)\right\rangle=\frac{1}{N} \sum_{i=1}^{N}\left\langle\left(r_{i}(t)-r_{i}(0)\right)^{2}\right\rangle$.

MSD can be used to characterize whether particle's motions are restricted to local regions or not.

\section{Results and discussions}

The MC $[7,8]$ and MD $[9,10]$ methods have already been used to study the phase behavior of colloidal systems with Yukawa potentials, in which the two-phase coexistence lines are determined utilizing the methods of Helmholtz free energy calculations and the Kofke integration [22]. The results indicate that BCC structure is thermodynamically stable for relatively small $\kappa$, but the dynamics of the crystallization were not discussed. However, the crystallization processes of charged colloidal suspensions are quite complex according to experimental observations in laboratory. Different crystalline structures are displayed by controlling the interactions of the particles [23], and even more the system ordering process may be inhibited to form glass phase [24,25]. So it is necessary to study the dynamics of the colloidal crystallization to further gain insight into the ordering process.

\subsection{The structure at small $\kappa$}

In this subsection, we focus on the case of soft interaction, i.e. the value of $\kappa$ is comparatively small. In this case, the inter-particle interactions are featured with long-range repulsion. Since the repulsion range varies with $\kappa$, for each pair of $\kappa$ and $\varepsilon$, there is a minimum particle number density that required for crystallization. This required particle number density can be quite small for the long-range repulsion. As a typical case, $\kappa=0.72, \varepsilon=120, \Phi=0.03$ were chosen in the simulation for the crystallization study. The simulation was started from a cubic box with particles randomly distributed. The results show that ordered BCC structures gradually emerge in different domains with time elapsing and eventually extend to all over the simulation box. The evolution of the RDF is illustrated by Fig. 2 .

From Fig. 3a we can see that, at the initial stage of the simulation, some peaks appear and the position of the first peak is 0.87 . However, the peak number is fewer and the heights of the peaks are rather low (except the first peak), which is consistent with the snapshot showing that the system is still in a disordered state. During the crystallization process, as shown in Fig. 3b-d, more peaks of the RDF line appear and the heights of the peaks increase obviously. Correspondingly, the snapshots show that some BCC structures appear in different parts of the simulation box. Some typical independent ordered parts in Fig. 3b with different orientations are displayed in Fig. 4.

With regard to the final structure after 250,000 time steps, the resulting RDF line shows obviously a lot of peaks, the positions of which fit the BCC crystal structure, as is shown in Fig. 3d. However, the peaks are not so sharp as RDF lines for perfect BCC structures, because the random force makes the particles vibrate around the lattice sites. The position of the first peak is 0.87 , which is consistent with the position of the first nearest neighbor of BCC structure and the value apparently remains unchanged from the beginning of the ordering process. According to the standard BCC structure, the second and the third peaks should be separated. However, these two peaks are so close that they are not well distinguishable in the RDF line. Actually, we can see that there is a split appears in the second peak of the RDF line, as shown in Fig. 3d, indicating that a highly ordered BCC structure has formed in the simulation after $t=0.5 \mathrm{~s}$. It has been reported that improper choice of particle number may mislead the resulted crystal structure due to the restriction of periodic boundary condition, especially when the simulation box is not large enough $[26,27]$. We purposely chose the particle number of $10,976\left(=4 \times 14^{3}\right)$ to match the number of lattice points for FCC structure in the simulation box. The simulation result that the particle number favorable for FCC structure still forms the BCC structure indicates that the latter is more stable. On the other hand, to match the BCC structure, the particle number should be $9826\left(=2 \times 17^{3}\right)$, as the closest choice for the cubic

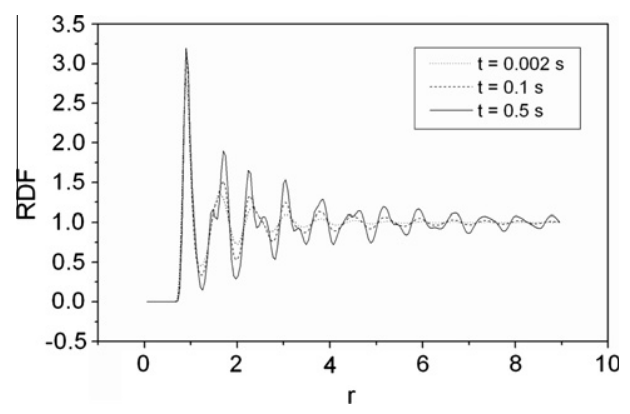

Fig. 2. Time-dependent change of RDF during the simulation at $\kappa=0.72, \varepsilon=120$, $\Phi=0.03$ for $N=10,976$ as a function of the scaled inter-particle distance $r$. Three lines are showed at $t=0.002 \mathrm{~s}, t=0.1 \mathrm{~s}, t=0.5 \mathrm{~s}$, respectively. See Fig. 3 for details. 
(a)

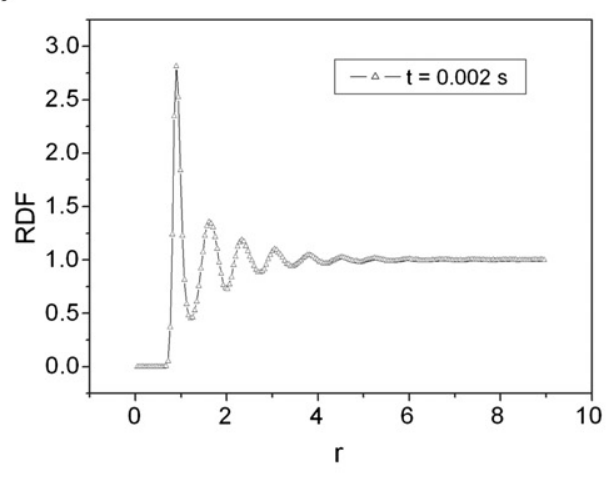

(b)

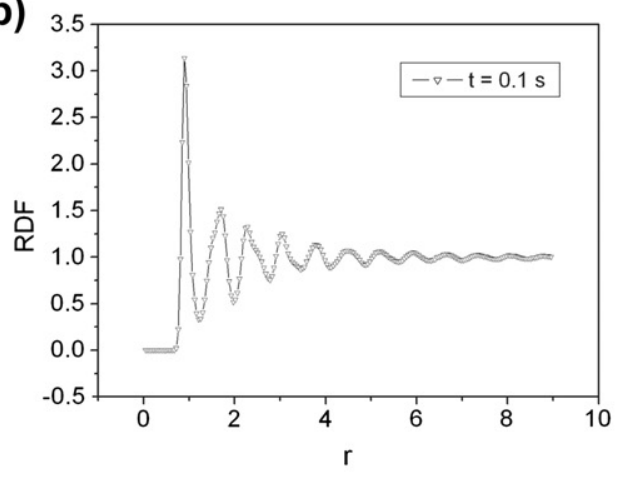

(c)

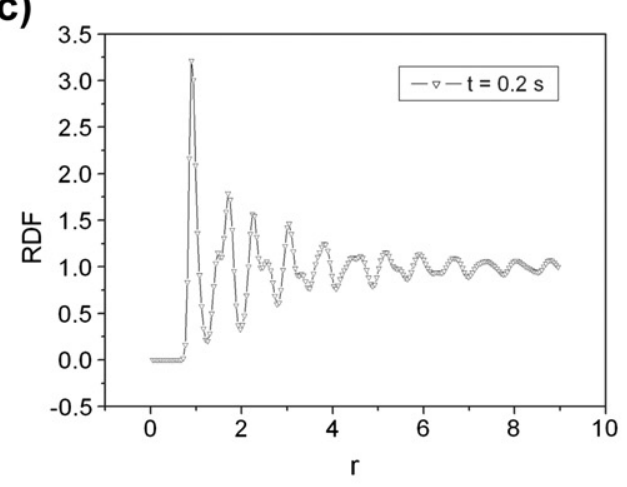

(d)

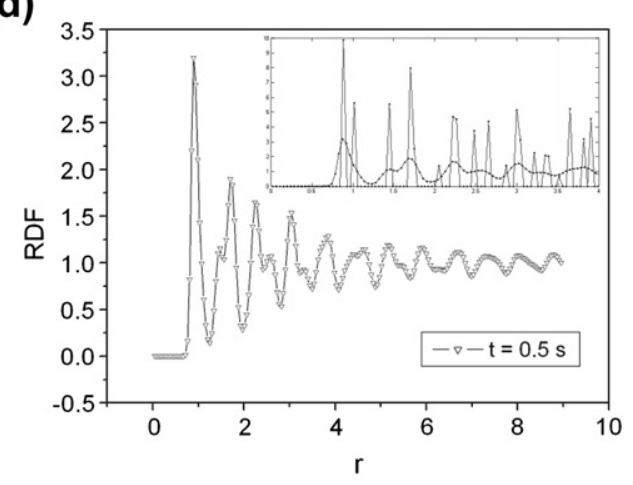

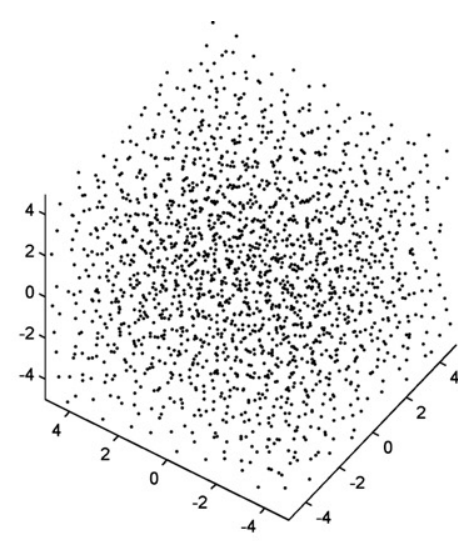
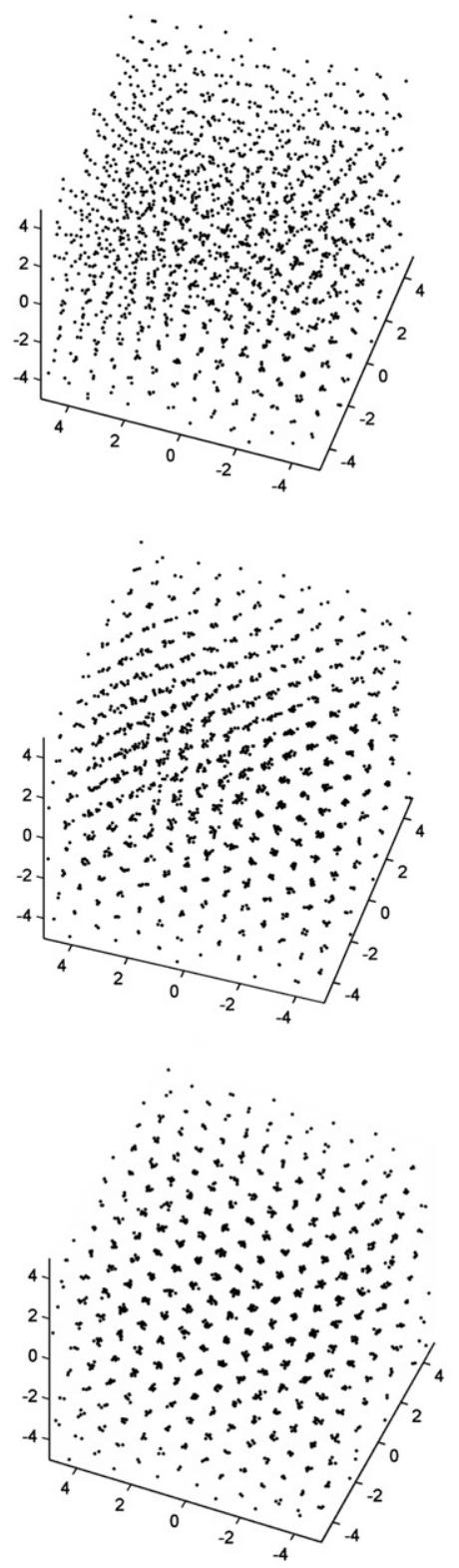

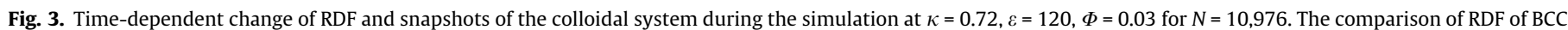

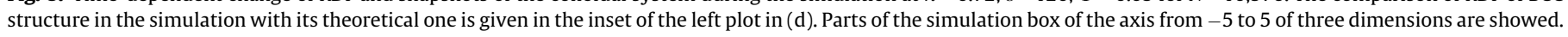

box. Since the particle number we choose is not right for BCC structure, some defects appear in the final BCC crystal arrays due to the presence of excess particles.
As the height of the first peak in RDF can be used to assess the extent of ordering, its evolutions are exhibited in Fig. 5. We can see that during the crystallization, the height of the first peak 

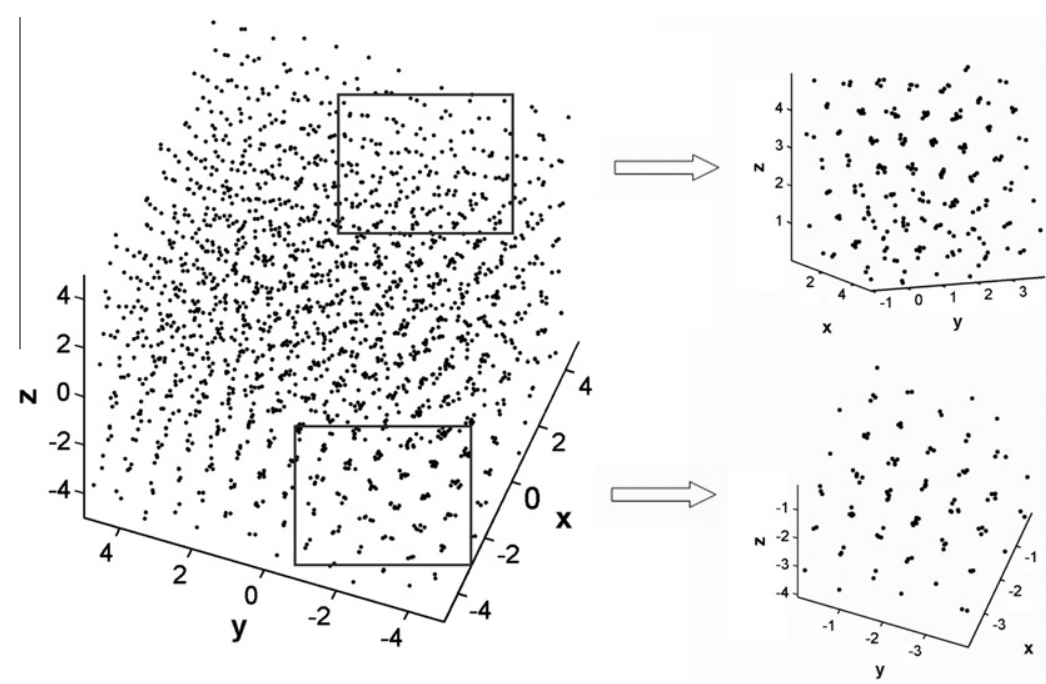

Fig. 4. Snapshots of typical independent ordered parts of the simulation box with different orientations, $t=0.1 \mathrm{~s}$.

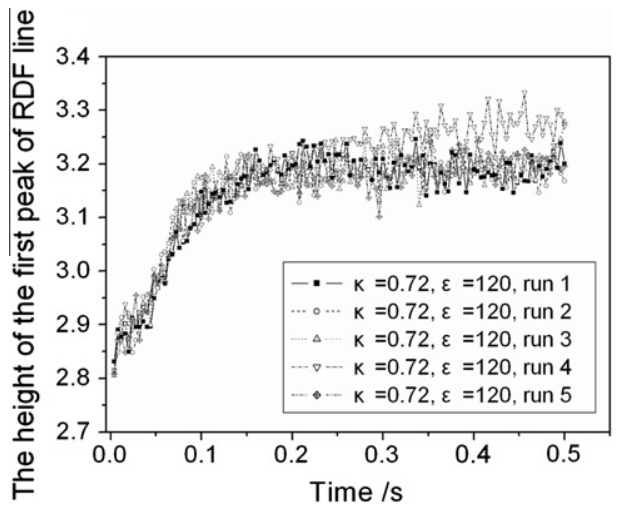

Fig. 5. The maximal height of the first peak of RDF line as a function of time, $\kappa=0.72, \varepsilon=120, \Phi=0.03, N=10,976$.

continues increasing with time, until it reaches a comparatively steady value after several thousands time steps $(t=0.20 \mathrm{~s})$, at which all over the simulation box is full of the BCC structures (see Fig. 3c). After $t=0.20 \mathrm{~s}$, the height of the first peak in RDF line fluctuates around a constant value as a result of the particles' vibration. The average height of the first peak is 3.2 from $t=0.20 \mathrm{~s}$ to $t=0.5 \mathrm{~s}$. The average is from five independent runs as shown in Fig. 5.

The change of MSD shown in Fig. 6 is consistent with the time evolution of the RDF line of Fig. 5. The MSD exhibits an obvious increase since the ordering process starts, and then reaches a plateau at $t=0.20 \mathrm{~s}$, which reflects the particles are moving only around their equilibrium positions with advancing crystallization. The average plateau value is 2.9 from $t=0.20 \mathrm{~s}$ to $t=0.5 \mathrm{~s}$, which is also sampled from the five independent runs.

\subsection{The structure evolution at large $\kappa$}

As mentioned above, the Yukawa potential of Eq. (1) with large $\kappa$ is close to the feature of the hard-sphere interaction. The formation of the FCC crystal structure in suspensions of hard-sphere colloids at high volume fraction was firstly predicted by computer simulations and then confirmed by experiments $[28,29]$. On the other hand, experimental studies on colloidal particles with repulsive interaction have also shown that the possibility of the formation of FCC crystal structures [3,22]. Actually, MC and MD simulations have

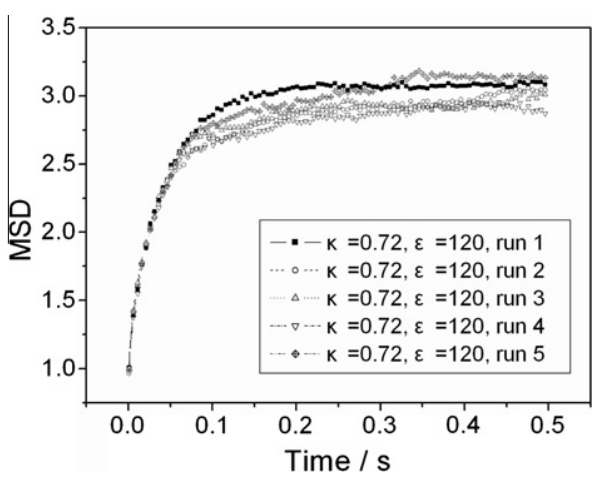

Fig. 6. MSD as a function of time, $\kappa=0.72, \varepsilon=120, \Phi=0.03, N=10,976$.

already verified that the FCC is a thermodynamically stable state for the systems featured with the Yukawa potential with large $\kappa$ [7-10]. As is well known, however, the thermodynamically favored structures are not necessarily dynamically directly reachable. Unfortunately, as a matter of fact, the dynamic evolution from liquid to the ordered FCC structure by computer simulations could hardly be actualized [10]. Extensive computational techniques, utilizing the classical nucleation theory and the free energy calculations, have been adopted to evaluate the nucleation rate and trace the crystallization process. It is argued that the nucleation probability is so small that the system could hardly crystallize spontaneously within a reasonable computation time. However, there remain discrepancies between the predictions of simulations and experiments [30,31].

In order to examine the dynamic possibility of FCC formation, BD simulations of colloidal particles interacting by the Yukawa potential with large $\kappa$ are implemented in this study. With large $\kappa$ corresponding to short-ranged repulsion, the required particle number density for crystallization has to be increased, compared with the case of small $\kappa$ discussed in previous subsection. The simulations with several different sets of $\kappa, \varepsilon$ and $\Phi$ have been carried out and the relevant simulation results are basically similar. One of them with parameters $\kappa=1.9, \varepsilon=50, \Phi=0.10$ are typically illustrated in Fig. 7. The cut-off distance of the interaction was set to be 2.175 with respect to the short-ranged repulsion. A number of long-run simulations demonstrate that the crystallization is basically impossible, and the particles are eventually jammed to form rather disordered structures before any tendency of crystallization 

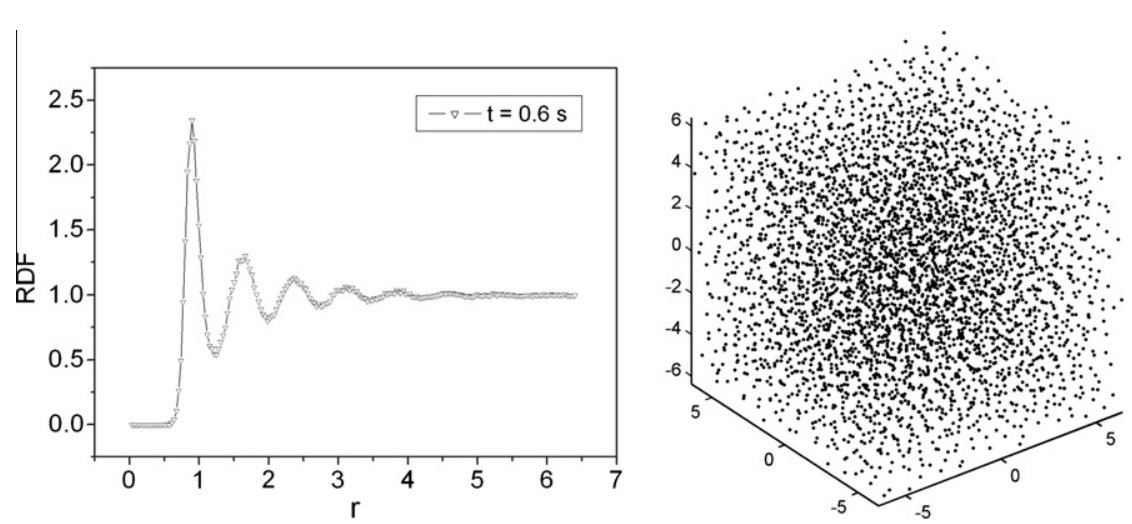

Fig. 7. Time-dependent change of RDF and snapshots of the system during the simulation at $\kappa=1.9, \varepsilon=50, \Phi=0.10$, for $N=4000$.

could show up. In this case, the particles are frozen and only oscillate locally with rather small amplitude. The result is displayed in Fig. 8, which shows that the RDF line does not change much from $t=0.01 \mathrm{~s}$ to $t=0.6 \mathrm{~s}$, and the average height of the first peak is around 2.3.

In Fig. 9 the time dependence of MSD is plotted. The MSD line reach its maximum at $t=0.3 \mathrm{~s}$, and the average value from $t=0.3 \mathrm{~s}$ to $t=0.6 \mathrm{~s}$ is 8.36 . This value remains the same for a long time, implying the system is no longer in the liquid state whose long-time MSD should grow linearly with time. Yet the system is still in a disordered state. Apparently, the colloidal particles are jumbled together and their motions are inhibited by the surrounding neighboring particles as in a glass state, which is shown in Fig. 7.

In fact, the colloidal model system has already been used to study the glass transition in experiments [31]. And it has also been observed that the interaction potential plays a determinant role in the system structure, as the charged colloidal suspension may get into glass or gel if the range of the repulsion is shortened at higher particle number density $[20,21]$.

\subsection{The transit from BCC to FCC at large $\kappa$}

It is shown in the previous subsection, that FCC structure is not directly accessible because particles are trapped into a stagnant disordered configuration. To examine whether the FCC structure is possible and which one would be more stable between BCC and FCC structures at large $\kappa$, instead of randomly distributed configuration, the BCC structure developed with the parameters described in subsection A was taken to be the initial configuration of the simulation. All the simulation parameters were kept the same as presented in

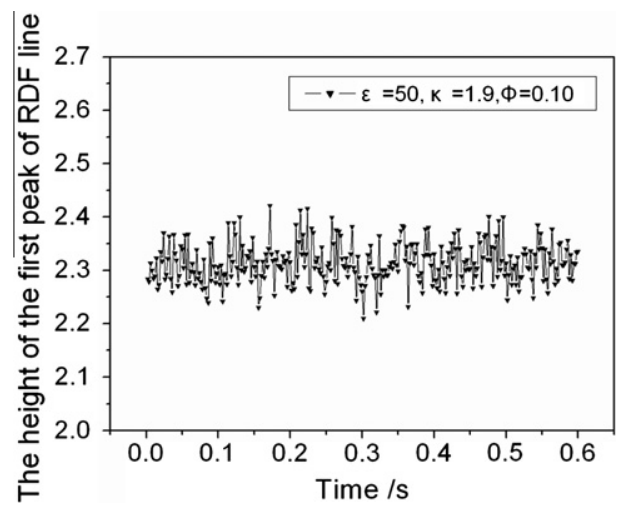

Fig. 8. The maximal height of the first peak of RDF line as a function of time, $\kappa=1.9$, $\varepsilon=50, \Phi=0.10$, for $N=4000$. the previous subsection, namely, $\kappa=1.9, \varepsilon=50, \Phi=0.10$, and the cut-off distance of the interaction was still set to be 2.175 . For this set of parameters, random displacements of the particles are much greater than displacements associated with the interaction between particles so that the transition from BCC structure to FCC structure becomes very slow. To accelerate the BCC-FCC transition process in the simulation, random displacements are reduced to 10 percent of their values in the calculation.

In the simulation, a transition from BCC to FCC structure can be detected according to the change of RDF in Fig. 10. The height of the first peak increases slightly as crystals of the FCC structure emerge, which is shown in Fig. 11. The average value of the height of the first peak from $t=0.1 \mathrm{~s}$ to $t=0.4 \mathrm{~s}$ is 7.34 . Meanwhile the position of the first peak moves to larger $r$, and the final position of the first peak is 0.91 . After 200,000 time steps, steady FCC structure is well established in the system, according to the peak distribution of RDF line which fits the FCC structure, as is shown in Fig. 10d.

As plotted in Fig. 12, the time dependence of MSD reach its maximum after $t=0.1 \mathrm{~s}$, and the average value from $t=0.1 \mathrm{~s}$ to $t=0.4 \mathrm{~s}$ is 0.94 .

Considering that the particle number $\left(4000=4 \times 10^{3}\right)$ used in the BCC-FCC transition exactly matches the number of lattice points for FCC structure, which may lead to the consequent FCC structure, the simulation with the initial particle number 9826 $\left(2 \times 17^{3}\right)$, which purposely matches the number of lattice points for BCC structure, was also carried out. In this case, BCC-FCC structural transition still occurred, further confirming the result that FCC phase is more stable than BCC, which is consistent with the phase diagram showing FCC is stable [7-10].

Our simulation shows that, although the FCC structure cannot form from liquid state as discussed in the previous subsection, a BCC-FCC transition can be actualized under the conditions that

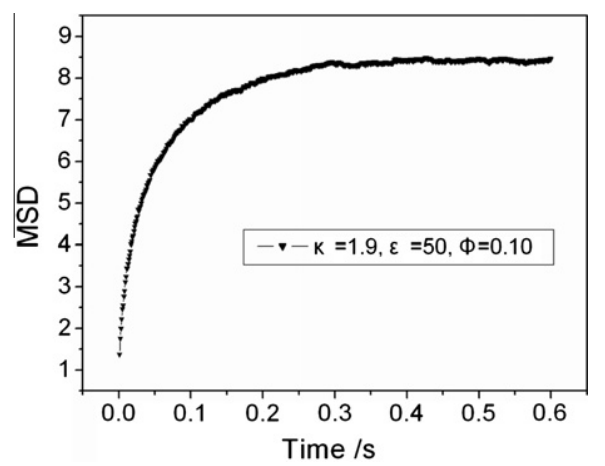

Fig. 9. MSD as a function of time, $\kappa=1.9, \varepsilon=50, \Phi=0.10$, for $N=4000$. 
(a)

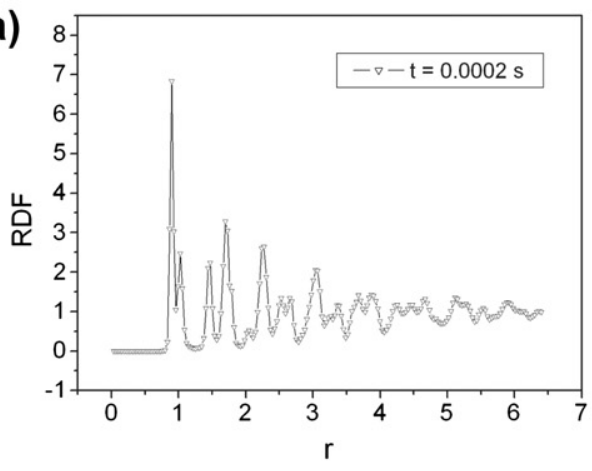

(b)

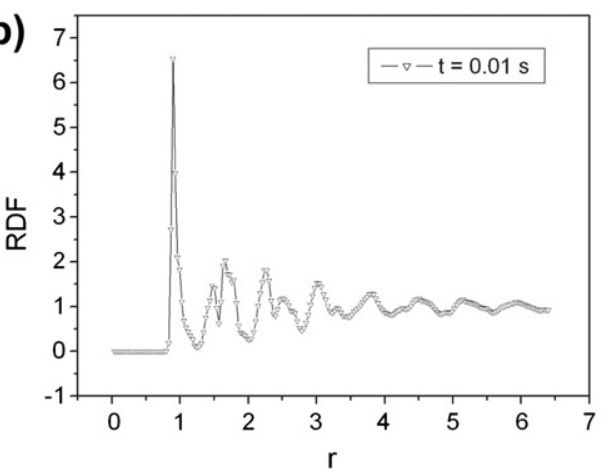

(c)

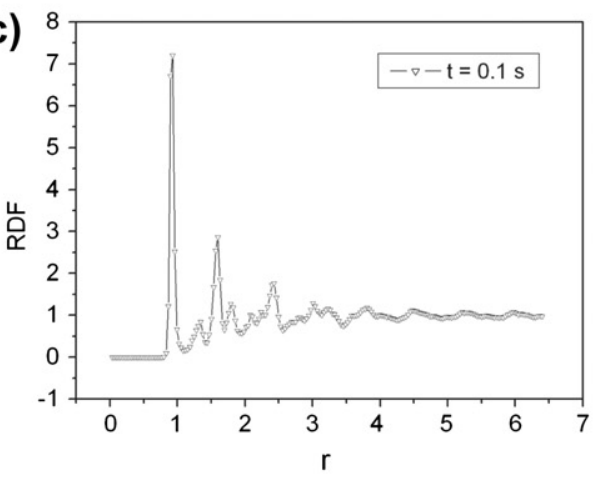

(d)

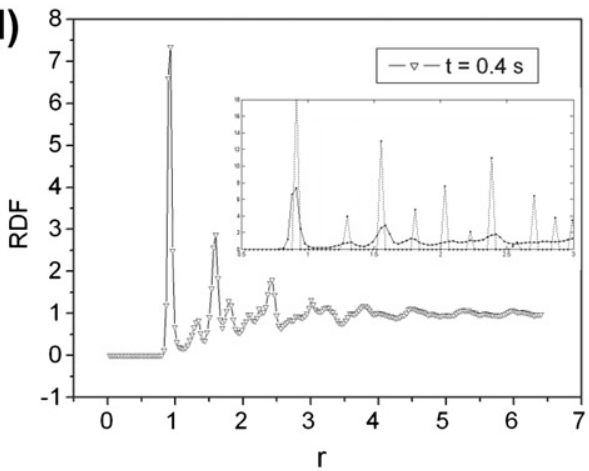

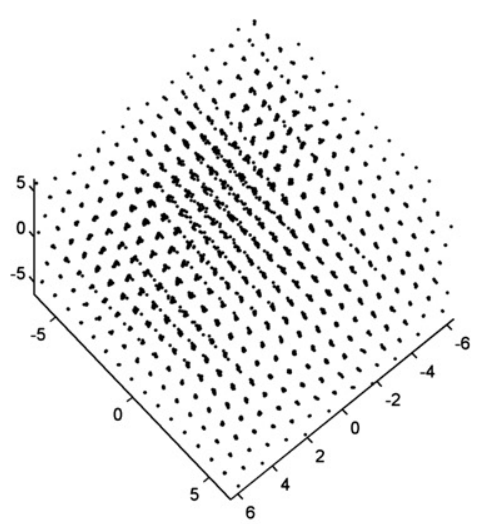

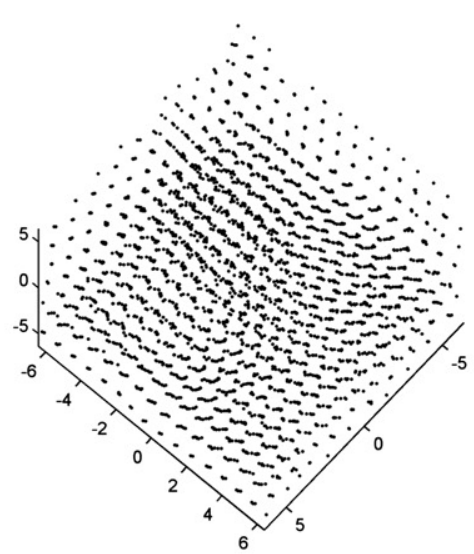

$\therefore$

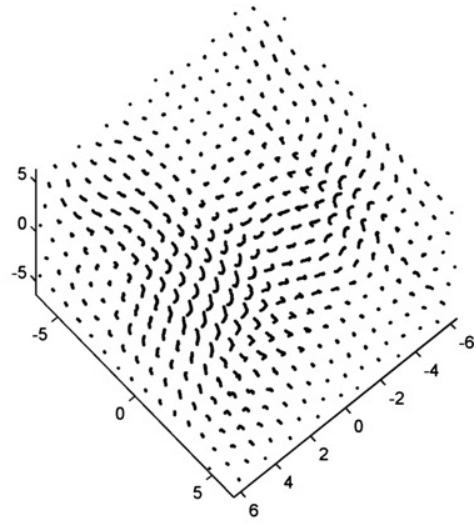

$\therefore \therefore$

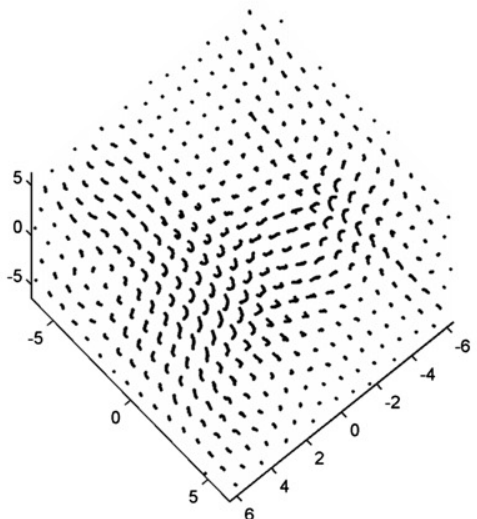

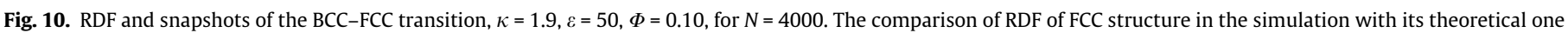
is given in the inset of the left plot in (d). 


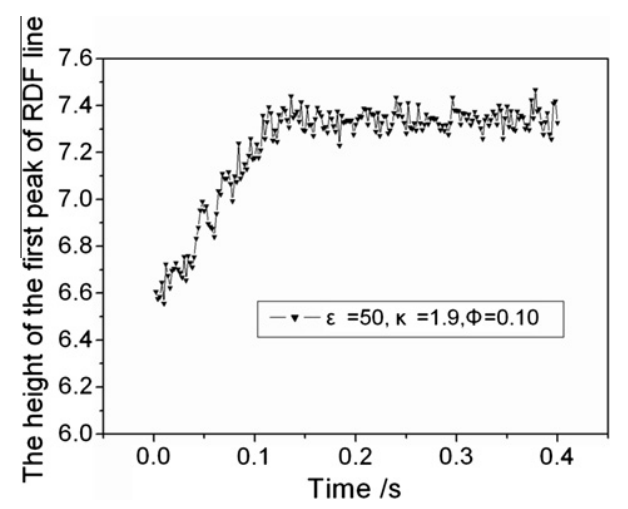

Fig. 11. The maximal height of the first peak of RDF line as a function of time, $\kappa=1.9, \varepsilon=50, \Phi=0.10$, for $N=4000$.

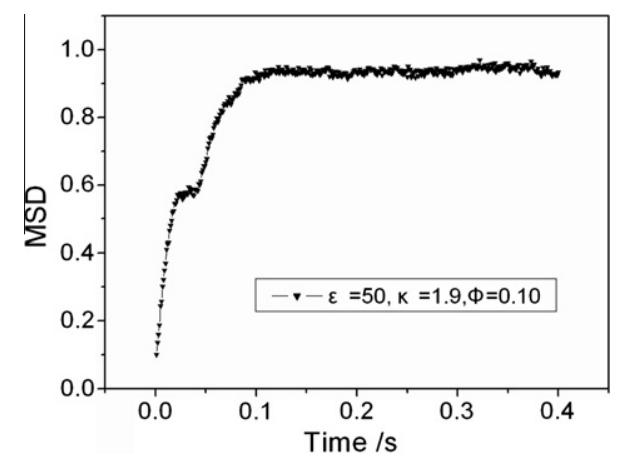

Fig. 12. MSD as a function of time, $\kappa=1.9, \varepsilon=50, \Phi=0.10$, for $N=4000$.

can make the FCC structure stable. From the results that the FCC structure is stable under certain interaction parameters and number densities but is not directly reachable from randomly distributed configurations, it can be reasonably inferred that there may exist an energy barrier between the disordered (liquid) state and ordered FCC structure. Thus the system will not crystallize unless the energy barrier is overcome or bypassed. This argument based on the simulation is in accordance with the predictions, according to the classical nucleation theory, showing that the spontaneous crystallization in short-ranged (large $\kappa$ ) and weakly-charged colloids is inaccessible within a reasonable time scale of computer simulation [5].

To alternatively examine whether FCC structure is more stable than the "glass state", we performed a simulation starting with a special configuration in which the particles in a small region of the simulation box were pre-arranged with FCC structure, while the particles in the rest part of space were in the glass state. During the simulation process we found that, boundaries of the FCC region were gradually expanding towards the glass domain, suggesting the FCC state is more stable than glass state.

According to all clues drawn from the simulation, for the case with large $\kappa$, a ranking of above-mentioned phases from most unstable to most stable are the disordered (liquid) state, the disordered glass state, BCC structure and FCC structure with a barrier between glass state and FCC.

\section{Conclusion}

In this paper, we present BD simulation to studying the dynamics of crystallization of charged colloidal system, in which the interaction between particles is approximated by the pair-additive repulsive Yukawa potential. The time evolution of crystallization process and the crystal structure during the simulation are de- tected by means of RDF and MSD. The simulations show that depending on the interaction parameters and particle number density, the system can be developed into one of the three possible states: to remain in liquid state; to be jammed in glass state or to form a stable crystal structure. The results in the manuscript are qualitatively in agreement with the phase diagram and the static results obtained by Chaikin et al. [32], which show that the solid state is BCC for long-range repulsions ( $\kappa$ is smaller) and FCC for short-range repulsions (large $\kappa$ ). The basic finding of this study can be summarized as two cases:

(I) When the inter-particle interactions are featured with longrange repulsion ( $\kappa$ is smaller), particles, at proper particle number densities, can spontaneously assemble into BCC arrays, but FCC structure is not stable.

(II) When particles have the short-ranged, hard-sphere-like interactions (large $\kappa$ ), the system either remains in liquid state (when particle number densities is low) or jammed to form disordered, glass state (at larger particle number densities). This glass state prevents the system from the crystallization possibility. When the glass state can be bypassed, FCC structure can be achieved and is more stable than BCC structure.

\section{Acknowledgments}

This work is supported by Grants 10672173 and 10972217 from the National Natural Science Foundation of China and the Knowledge Innovation Program of the Chinese Academy of Sciences (including Grant No. KJCX2-YW-L08).

\section{References}

[1] C.A. Murray, D.G. Grier, Annu. Rev. Phys. Chem. 47 (1996) 421.

[2] William B. Russel, Nature 421 (2003) 490.

[3] M.E. Leunissen, A.P. Hynninen, J. Chem. Phys. 124 (2006) 244706

[4] J.A. Weiss, A.E. Larsen, D.G. Grier, J. Chem. Phys. 109 (1998) 8659.

[5] S. Auer, D. Frenkel, J. Phys.: Condens. Matter. 14 (33) (2002) 7667.

[6] U. Gasser, E.R. Weeks, A. Schofield, P.N. Pusey, D.A. Weitz, Science 292 (2001) 258.

[7] F. El Azhar, M. Baus, J.P. Ryckaert, E.J. Meijer, J. Chem. Phys. 112 (2000) 5121.

[8] A.P. Hynninen, M. Dijkstra, Phys. Rev. E 68 (2003) 021407.

[9] K. Kremer, M.O. Robbins, G.S. Grest, Phys. Rev. Lett. 57 (1986) 2694.

[10] M.O. Robbins, K. Kremer, G.S. Grest, J. Chem. Phys. 88 (1988) 3286.

[11] M. Ishikawa, H. Morimoto, T. Maekawa, J. Cryst. Growth 237 (2002) 1825.

[12] D.L. Ermak, J.A. McCammon, J. Chem. Phys. 69 (1978) 1352.

[13] S.H. Xu, Z.W. Sun, J. Chem. Phys. 126 (2007) 144903.

[14] A.P. Hynninen, M. Dijkstra, J. Phys.: Condens. Matter. 15 (2003) S3557.

[15] M. Brunner, J. Dobnikar, H.H. von Grunberg, C. Bechinger, Phys. Rev. Lett. 92 (2004) 078301.

[16] Z.W. Sun, S.H. Xu, J. Liu, Y.M. Li, L.R. Lou, J.C. Xie, J. Chem. Phys. 122 (2005) 184904.

[17] Z.W. Sun, S.H. Xu, G.L. Dai, Y.M. Li, L.R. Lou, Q.S. Liu, R.Z. Zhu, J. Chem. Phys. 119 (2003) 2399.

[18] C. Russ, R. van Roij, M. Dijkstra, H.H. von Grunberg, Phys. Rev. E 66 (2002) 011402.

[19] J.P. Hansen, L. Verlet, Phys. Rev. 184 (1969) 151.

[20] F.H. Stillinger, T.A. Weber, Phys. Rev. B 22 (1980) 3790.

[21] P.J. Steinhardt, D.R. Nelson, M. Ronchetti, Phys. Rev. B 28 (1983) 784-805.

[22] D.A. Kofke, J. Chem. Phys. 98 (1993) 4149.

[23] M.A. Bevan, J.A. Lewis, P.V. Braun, P. Wiltzius, Langmuir 20 (2004) 7045.

[24] D. Frenkel, Science 296 (2002) 65.

[25] L. Liu, S.H. Xu, J. Liu, L. Duan, Z.W. Sun, R.X. Lu, P. Dong, Acta Phys. Sin. 55 (2006) 6168.

[26] J.D. Honeycutt, H.C. Andersen, Chem. Phys. Lett. 108 (1984) 535.

[27] J.S. Vanduijneveldt, D. Frenkel, J. Chem. Phys. 96 (1992) 4655.

[28] W.G. Hoover, F.H. Ree, J. Chem. Phys. 49 (1968) 3609.

[29] P.N. Pusey, W. Vanmegen, P. Bartlett, B.J. Ackerson, J.G. Rarity, S.M. Underwood, Phys. Rev. Lett. 63 (1989) 2753.

[30] V.J. Anderson, H.N.W. Lekkerkerker, Nature 416 (2002) 811.

[31] J. Lodge, D. Heyes, Phys. Chem. Chem. Phys. 1 (1999) 2119.

[32] S.K. Sinha, P.M. Chaikin, J.D. Axe, Y. Fujii, Phys. Rev. Lett. 62 (1989) 1524. 